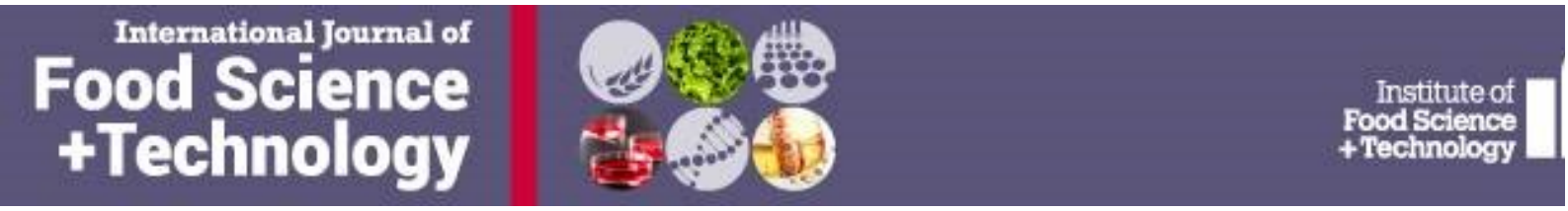

\title{
Production of flavonoid and lignan aglycones from flaxseed and soy extracts by Bifidobacterium strains
}

\begin{tabular}{|c|c|}
\hline Journal: & International Journal of Food Science and Technology \\
\hline Manuscript ID & Draft \\
\hline Manuscript Type: & Original Manuscript \\
\hline $\begin{array}{r}\text { Date Submitted by the } \\
\text { Author: }\end{array}$ & $\mathrm{n} / \mathrm{a}$ \\
\hline Complete List of Authors: & $\begin{array}{l}\text { Peiroten, Angela; Instituto Nacional de Investigacion y Tecnologia } \\
\text { Agraria y Alimentaria, Food Technology } \\
\text { Álvarez, Inmaculada; ICTAN, USTA } \\
\text { Landete, José Maria; Instituto Nacional de Investigacion y Tecnologia } \\
\text { Agraria y Alimentaria, Food Technology }\end{array}$ \\
\hline Keywords: & $\begin{array}{l}\text { Soybeans, Flaxseed, Bifidobacterium, Quercetin, Herbacetin, } \\
\text { Quercetagetin }\end{array}$ \\
\hline
\end{tabular}

\section{SCHOLARONE ${ }^{\text {TM }}$ \\ Manuscripts}



Bifidobacterium strains

5

6 Departamento de Tecnología de Alimentos, Instituto Nacional de Investigación y

7 Tecnología Agraria y Alimentaria (INIA). Carretera de La Coruña Km 7.5, 28040.

$8 \quad$ Madrid (Spain)

$9{ }^{2}$ Unidad de Servicio de Técnicas Analíticas, Instrumentales y Microbiología (USTA),

10 Instituto de Ciencia y Tecnología de los Alimentos y Nutrición (ICTAN-CSIC), José

11 Antonio Novais, 10, 28040 Madrid, (Spain)

12

13

14 *Address correspondence to José M Landete, landete.josem@inia.es 


\section{Abstract}

20 Flaxseed and soybean have are an important source of phytochemical compounds, 21 mainly, lignans and isoflavones, but also of flavones, flavanones and flavonols. These 22 compounds appear mainly glycosylated in plant food, and must be converted into 23 aglycones to increase their bioavailability. In this work we analyse the ability of 24 Bifidobacterium strains $(\mathrm{n}=25)$ to produce lignan and flavonoids aglycones from 25 flaxseed and soybean extracts. Most of the Bifidobacterium strains increased the 26 concentrations of secoisolariciresinol, daidzein, genistein, naringenin, eriodyctiol, 27 luteolin and apigenin. Moreover, Bifidobacterium pseudocatenulatum and 28 Bifidobacterium breve strains showed high production of herbacetin, increased the 29 kaempferol concentration and produced quercetin and quercetagetin. $B$. 30 pseudocatenulatum INIA P815 and B. breve INIA P367 produced $32.37 \pm 2.81$ and $3128.64 \pm 3.36 \mathrm{mg}$ respectively of herbacetin/g of lignan extract. Bifidobacterium strains 32 transformed the glycosides of a wide range of flavonoids into their aglycones, 33 increasing the antioxidant activity and improving their bioavailability.

35 Keywords: herbacetin; quercetagetin; quercetin; naringenin; kaempferol; 36 Bifidobacterium; soy; flaxseed. 
37

38

39

40

41

42

\section{Introduction}

Flavonoids and lignans are phenolic compounds present in many plant foods. Flavonoids are a diverse group of phytonutrients that encompasses several groups: anthocyanidins, flavanols, flavones, flavonols, flavanones and isoflavones. Although all these compounds are minor components of food, each type of flavonoids and lignans carries its own distinct set of actions and benefits for human health. Many studies have suggested the protective role of flavonoids in cardiovascular disease due to their antioxidant properties, which could also have influence in other diseases as cancer and neurodegenerative diseases (Airoldi, La Ferla, Orazio, Ciaramelli, \& Palmioli, 2018; Zamora-Ros, Touillaud, Rothwell, Romieu, \& Scalbert, 2014). Moreover, isoflavones and lignans are classified as phytoestrogens, compounds with structural similarity to estrogen and a weak affinity for the estrogen receptor (Vaya \& Tamir, 2004). Phytoestrogen intake has been associated with the amelioration of menopausal symptoms and with decreased risks of breast and prostate cancer, cardiovascular disease and diabetes (Rietjens, Louisse, \& Beekmann, 2017).

Flavonoids and lignans appear in plant foods in the form of $\mathrm{O}$ - or $\mathrm{C}$-glycosides that are not absorbed by the intestinal epithelium and must be firstly transformed into aglycones (Landete, Arques, Medina, Gaya, de Las Rivas, \& Muñoz, 2016). The deglycosylation is carried out in our organism by the intestinal microbiota, which produce the aglycones and can further transform them into other bioactive compounds. The aglycones show higher bioavailability and antioxidant and estrogenic/antiestrogenic activities and thus it has been suggested that the beneficial effects of polyphenols could be exerted by their aglycones (Peirotén, Bravo, \& Landete, 2019a). 
61 Among the food products rich on phenolic compounds, flaxseed and soybean have been

62

63

64

studied for being particularly rich in lignans and isoflavones respectively. Flaxseed (Linum usitatissimum L.), one of the oldest cultivated crops, continues to be widely grown for oil, fibre and food (Oomah, 2001). Flax or flaxseed oil are used as ingredients in baked foods, dairy products, milk, juices, dry pasta and meat products (Goyal, Sharma, Upadhyay, Gill, \& Sihag, 2014). There is an increasing interest in the health effect of flaxseed and flax oil consumption due to its high content in biologically active components, such as alpha-linolenic acid and lignans. Soybean (Glycine max) is a legume widely cultivated for its multiple uses in food, feed and other industries (McClure, Cocuron, Osmark, McHale, \& Alonso, 2017). In addition, soy is of great interest for human health due to its high content in isoflavones. Both soybean and flaxseed contain also other groups of phenolic compounds, including several types of flavonoids (Herchi, Arraez-Roman, Trabelsi, Bouali, Boukhchina, Kallel, et al., 2014; Landete, Hernandez, Robredo, Duenas, de las Rivas, Estrella, et al., 2015).

In previous works we described how most Bifidobacterium strains were able to produce the lignan aglycone secoisolariciresinol (SECO) from secoisolariciresinol diglucoside (SDG) (Peirotén, Gaya, Álvarez, Bravo, \& Landete, 2019b), which is the main lignan in flaxseed (Toure \& Xu, 2010). Bifidobacterium strains are also able to produce the isoflavone aglycones daidzein and genistein (Gaya, Peirotén, Medina, \& Landete, 2016b). The ability of bifidobacteria to deglycosilate a wide range of substrates seem to be linked to a variety of $\beta$-glycosidases encoded in their genome (Guadamuro, Flórez, Alegría, Vázquez, \& Mayo, 2017). In this work, we explored if the ability of Bifidobacterium strains to deglycosilate lignans and isoflavones also applies to other flavonoids, such as flavanones, flavones, flavanols and flavonols. We chose a broth enriched with flaxseed and soy extracts as a model of a food rich in a diversity of 
86 phenolic compounds. A selection of 25 Bifidobacterium strains belonging to ten

87 different species was chosen for the fermentations, after which the content in specific

88 aglycones, the antioxidant activity and the total phenolic compounds were determined.

Material and methods

92

\section{Bacterial growth conditions}

94

The 25 Bifidobacterium strains tested in this study (Table 1) were selected from the

INIA culture collection based on their biotechnological and probiotic properties. The Bifidobacterium were cultivated under strict anaerobic conditions at $37^{\circ} \mathrm{C}$ in $\mathrm{RCM}$ broth (BD, Le Pont de Claix, France) and subcultured twice before using them in the 99 fermentation experiments.

\section{Fermentation of flaxseed and soy extracts by Bifidobacterium strains}

Flaxseed extract (LinumLifeTM EXTRA) and soy extract (SoyLifes EXTRA) were 104 purchased from Frutarom Netherlands BV (Veenendaal, The Netherlands) and initially 105 dissolve in DMSO (Sigma Aldrich, Poole, UK). BHI medium (Biolife, Milan, Italy) 106 containing $0.5 \mathrm{~g} / \mathrm{L}$ of L-cysteine was supplemented with flax seed extracts $(2 \mathrm{~g} / \mathrm{L})$ and 107 soy extracts $(4 \mathrm{~g} / \mathrm{L})$ and the $\mathrm{pH}$ was adjusted to 7.4 with $\mathrm{NaOH}$. Ten $\mathrm{mL}$ of these 
enriched media was inoculated with the Bifidobacterium strains. The residual amount of DMSO present in the media was checked to not interfere with bacterial growth. The inoculated broths were incubated in sealed jars under anaerobic conditions using AnaeroGen sachets (Oxoid Ltd., Basingstoke, United Kingdom) at $37{ }^{\circ} \mathrm{C}$ for 5 days. Non-inoculated broths containing flaxseed and soy extracts were used as controls. The experiments were carried out in triplicate.

\section{Extraction of flavonoids and lignans}

After the incubation of the broths containing soy and flaxseed extracts, the extraction of flavonoids and lignans was performed twice with $2 \mathrm{~mL}$ of diethyl ether, and twice with $2 \mathrm{~mL}$ of ethyl acetate according to Gaya, Arqués, Medina, Álvarez, and Landete (2016a). The solvents were evaporated at room temperature under an $\mathrm{N}_{2}$ stream, and the residue was dissolved in $300 \mathrm{~mL}$ methanol/water $(50: 50, \mathrm{v} / \mathrm{v})$. The extracts were filtered through a $0.22 \mathrm{~mm}$ cellulose acetate filter (Millipore, Madrid, Spain) before transferring them to HPLC vials and storing them at $-20^{\circ} \mathrm{C}$.

\section{Flavonoids and lignans analysis by HPLC-ESI/MS and HPLC-ESI/MS/MS.}

Flavonoids and lignans were analysed by HPLC-ESI/MS and HPLC-ESI/MS/MS as previously described (Gaya, et al., 2016a; Gaya, Peirotén, Álvarez, Medina, \& Landete, 2018). Briefly, separation of phenolic compounds was achieved on a reverse phase Nova-Pak C18 column (300 x 3.9 mm, $4 \mu \mathrm{m})$ (Waters, Barcelona, Spain). A volume of 
$13125 \mu \mathrm{L}$ was injected and elution was performed with a gradient consisting of solvent $\mathrm{A}$ 132 (water/acetic acid, 98:2 v/v), solvent B (water/acetonitrile/acetic acid, 78:20:2 v/v/v) 133 and solvent $\mathrm{C}$ (acetonitrile) at a flow rate of $1 \mathrm{~mL} / \mathrm{min}$. Analyses were carried out using 134 an Agilent 1200 series LC, comprised of quaternary pump with integrated degasser, 135 thermostated autosampler, thermostated column compartment and diode array detector, 136 coupled with an Agilent 6530 Accurate-Mass Quadrupole Time of Flight (Q-TOF) 137 LC/MS with ESI-Jet Stream Technology (Agilent Technologies, Waldbroon, Germany). 138 The Q-TOF acquisition method was $4 \mathrm{GHz}$, mass range low $1700 \mathrm{~m} / \mathrm{z}$, negative polarity, 139 drying gas $10 \mathrm{l} / \mathrm{min}$ and $350^{\circ} \mathrm{C}$, sheath gas $11 \mathrm{l} / \mathrm{min}$ and $350^{\circ} \mathrm{C}$, nebulizer 45 psi, 140 capillary voltage $3500 \mathrm{~V}$, nozzle voltage $0 \mathrm{~V}$ and fragmentor voltage $120 \mathrm{~V}$. For the 141 identification and characterization of flavonoids MS/MS experiments were performed 142 with 20V collision energy. Data acquisition (version B.05.01) and Qualitative Analysis 143 (version B.07.00) of MassHunter Workstation Software were used (Agilent 144 Technologies).

145 Quantification was done by means of external standard calibration curves, and the data 146 expressed as $\mathrm{mg}$ of compound per litre of fermentation media. The different 147 concentration of standard compounds were added to BHI medium and subsequently 148 extracted and analysed as above mentioned. SECO, naringenin, eriodyctiol, hesperetin, 149 luteolin, apigenin, vitexin, catechin, epicatechin, kaempferol, kaempferol-3-O150 rutinoside, quercetin, isoquercetin, rutin, taxifolin and mirecitin, were purchased from 151 Sigma-Aldrich, whereas herbacetin and quercetagetin were purchased from 152 Extrasynthese (Genay, France) and daidzein, daidzin, genistein and genistin were 153 purchased from LC Laboratories (Woburn, MA). 
157 Antioxidant activity was determined in triplicate by the ferric reducing antioxidant 158 power (FRAP) method according to del Olmo, Picón, and Núñez (2018). An aliquot of $15920 \mu \mathrm{l}$ of supernatants were incubated at $37^{\circ} \mathrm{C}$ for $60 \mathrm{~min}$ with $100 \mu \mathrm{l}$ of Milli-Q water 160 and $900 \mu \mathrm{l}$ of the FRAP reagent containing 2,4,6-tris(2-pyridyl)-s-triazine (TPTZ, 161 Sigma-Aldrich) and $\mathrm{FeCl}_{3} 6 \mathrm{H}_{2} \mathrm{O}$. The absorbance was read at $595 \mathrm{~nm}$ to monitor $\mathrm{Fe}^{3+}$ 162 reduction and the formation of a colored TPTZ-Fe ${ }^{2+}$ complex. A standard curve was obtained using Trolox (Sigma-Aldrich) to allow quantification.

164 Phenolic compounds were determined in triplicate by the Folin-Ciocalteau colorimetric method adapted from Ferraces-Casais, Lage-Yusty, Rodríguez-Bernaldo de Quirós, and López-Hernández (2012). The fermented broths $(100 \mu \mathrm{L})$ were mixed with a $6 \%$ $\mathrm{Na}_{2} \mathrm{CO}_{3}$ aqueous solution $(1 \mathrm{~mL})$ and the Folin-Ciocalteau reagent $(100 \mu \mathrm{L})$. The mixture was incubated for $1 \mathrm{~h}$ at room temperature and the absorbance was read at 740 nm. Quantification was carried out using a gallic acid (Sigma Aldrich) standard curve.

Results

\section{SECO production by Bifidobacterium strains}

176 SECO was detected in all the fermented broths, in levels higher than the control for the majority of the Bifidobacterium strains. The three Bifidobacterium pseudocatenulatum strains and two of the Bifidobacterium breve strains (INIA P367, INIA P776) produced 179 the highest SECO concentrations (Table 2). These five strains produced concentrations of SECO higher than $100 \mathrm{mg} / \mathrm{L}$, equivalent to $50 \mathrm{mg}$ per gram of flaxseed extracts. 


\section{Isoflavone aglycones production by Bifidobacterium strains}

183

The majority of Bifidobacterium strains analysed were able to convert the daidzin and genistin to their aglycones daidzein and genistein (Table 2). However, large differences in aglycone production were observed between strains. The higher levels of combined daidzein and genistein production were found in the three $B$. breve strains. $B$. pseudocatenulatum strains also showed high concentrations of the aglycones, and $B$. pseudocatenulatum INIA P846, and B. pseudocatenulatum INIA P946 also showed the genistein. strains and Bifidobacterium catenulatum INIA P825 (Table 2).

\section{Flavanone aglycones produced by Bifidobacterium strains}

201

202 An increase in the naringenin concentration was observed as a result of the metabolism 203 of most of the Bifidobacterium strains (Table 4) (Figure S1). The B. breve, $B$. pseudocatenulatum and B. catenulatum strains, together with B. dentium INIA P886 and 
205 B. animalis INIA P913, showed the highest levels, with a 5 fold increase over the

206

207 Eriodictyol showed a slight increase compared to control as a result of the microbial 208

212

213

214

215

216

217

218

219

220

221

222

223

224

225

226

227

228

229 metabolism of most of the Bifidobacterium (Figure S1). On its part, hesperetin was not detected either in the controls or as a product of the microbial metabolism (Table 4).

\section{Flavone aglycones produced by Bifidobacterium strains}

Luteolin was found in the all samples analysed (Figure S1), showing some bifidobacterial strains small increases compared to control (Table 3). Interestingly, Bifidobacterium infantis INIA P728 degraded most of the luteolin present in the broth. Apigenin, not detected in the control broth, appeared as a consequence of the metabolism of most of the Bifidobacterium strains (Table 3) (Figure S1). B. breve INIA P12, B. breve INIA P776, B. longum INIA P750, B. pseudocatenulatum INIA P846 and B. pseudocatenulatum INIA P946 were the strains with the highest apigenin production. Vitexin, an apigenin flavone glucoside was detected in all the samples (Table 4), with no apparent variation due to the microbial metabolism (data not shown).

\section{Flavanol aglycones}

Catechin and epicatechin were not detected either in the control or as a result of the fermentation by the Bifidobacterium strains (Table 4).

\section{Flavonol aglycones produced by Bifidobacterium strains}


230 The flavonol aglycone found in higher concentrations was herbacetin (Table 3). It was 231 not present in the control and was detected in 15 of the Bifidobacterium strains. The 232 highest levels of herbacetin were produced by the three $B$. breve strains, $B$. 233 pseudocatenulatum INIA P815 and B. pseudocatenulatum INIA P946. Two compounds 234 present in the control sample were tentatively identified as glycosides of herbacetin 235 (Table 4). Herbacetin diglucoside concentrations decreased in those fermented broths in 236 which herbacetin was detected, and was totally consumed by B. pseudocatenulatum 237 INIA P815, the strain showing the highest herbacetin concentration.

238 A compound with equal molecular mass to that of herbacetin was detected and 239 identified as quercetin. Herbacetin and quercetin were identified by means of 240 comparison with the standard compounds (Figure 1A) and were confirmed by MS/ MS 241 (Figure 1B).

242 Quercetin was detected in low levels in four of the broths fermented by bifidobacteria, 243 corresponding to $B$. breve INIA P367, B. breve INIA P776, B. pseudocatenulatum INIA 244 P815 and B. pseudocatenulatum INIA P946 (Table 3). Of the two quercetin glycosides 245 studies, rutin was not detected in any sample while isoquercetin, was identified in 246 almost all of them. Isoquercetin was completely metabolized by B. pseudocatenulatum 247 INIA P815, and its levels decreased when $B$. animalis INIA P490, the two B. dentium, 248 the three B. breve and the remaining B. pseudocatenulatum strains fermented the broths. 249 Nevertheless, quercetin, the expected result of isoquercetin deglycosilation, was 250 detected only in some of those strains.

251 Similarly to quercetin, the aglycone quercetagetin (Figure 2) was not found in the 252 control and only in a few of the bifidobacteria-fermented broths, namely in $B$. 253 pseudocatenulatum INIA P946, B. pseudocatenulatum INIA P815, the three B. breve 
254 strains and B. dentium INIA P886 (Table 3). Finally, the other flavonol aglycones

255 tested, mirecitin and taxifolin, were not detected in any sample.

256 Kaempferol was found in low concentrations in control samples and not all the

257 Bifidobacterium strains were able to increase its levels (Table 3) (Figure 1S). The three

258 B. breve strains, B. pseudocatenulatum INIA P815, B. pseudocatenulatum INIA P946

259 and $B$. dentium INIA P886 were the strains that produced the highest concentration of

260 kaempferol, with a tenfold increase over the control. The glycoside kaempferol-3-O-

261 rutinoside was not found in any of the samples.

264 Antioxidant activity and phenolic compounds

265 A small increase in antioxidant activity was observed for all the Bifidobacterium strains

266 compared with the control broth with flaxseed and soy extracts. The highest increases

267 were found in the broths fermented by $B$. breve INIA P367, $B$. breve INIA P776, $B$.

268 dentium INIA P886 and B. pseudocatenulatum INIA P946 (Table 5).

269 The determination of phenolic compounds also showed moderate increments in the 270 broths fermented with Bifidobacterium strains, although not all of them were superior to 271 control. B. breve INIA P367 and B. catenulatum INIA P825 resulted in the highest reads 272 for phenolic compounds.

Discussion 
277 For years, soy and flaxseed and have received attention because of their phytoestrogen 278 content of isoflavones and lignans respectively. As seen in this and other works, both 279 foods contain also other flavonoids (Correa, Li, Aldini, Carini, Oliver Chen, Chun, et 280 al., 2010; Herchi, et al., 2014; Landete, et al., 2015). All these compounds are present 281 in plant food as glycosides and must be metabolized into aglycones to increase their 282 bioavailability. Given their estrogenic nature, many studies have addressed the effect of 283 lignan and isoflavones, such as SECO, daidzein and genistein, in menopausal symptoms and the prevention of breast cancer. Moreover, their effect in the lowering of risk of cardiovascular disease, metabolic syndrome and other cancers has been also described (Peirotén, et al., 2019a; Rietjens, et al., 2017; Sirotkin \& Harrath, 2014). The effect of other flavonoids, such as flavanones, flavones and flavonols has been less studied. Quercetin, kaempferol, naringenin and eriodictyol exhibit antioxidant and antiinflammatory effects, which confers them potential beneficial effects in preventing different inflammation-related diseases (Devi, Malar, Nabavi, Sureda, Xiao, Nabavi, et al., 2015; Egert, Bosy-Westphal, Seiberl, Kürbitz, Settler, Plachta-Danielzik, et al., 2009; Lou, Jing, Ren, Wei, \& Zhang, 2012; Narváez-Mastache, Novillo, \& Delgado, 2008; Venkateswara, Kiran, Rohini, \& Bhagyasree, 2017). Herbacetin has been described to have anti-hyperglycaemic and anti-hyperlipidemic properties (Veeramani, Alsaif, \& Al-Numair, 2018). Quercetagetin exhibited strong in vitro antioxidant, antidiabetic and antilipidemic activities, having potential application for the treatment of 297 obesity and diabetes (Wang, Xu, Chen, Tai, Liu, \& Gao, 2016).

In previous works, Bifidobacterium strains has shown the ability to deglycoside SGD, the main lignan in flaxseed, into SECO (Peirotén, et al., 2019b), and to transform the isoflavones daidzin and genistin into their aglycones daidzein and genistein (Gaya, et $a l ., 2016 \mathrm{~b})$. In agreement with that, the fermentation of a mixture of flaxseed and soy 
302 extracts by the 25 Bifidobacterium strains tested resulted in the production of the

303

304

305

306

307

308

309

310

311

312

313

314

315

316

317

318

319

320

321

322

323

324

325

326 aglycones SECO, daidzein and genistein.

Alongside isoflavones and lignans, the flaxseed and soy extracts contain a series of other flavonoid glycosides, which can be deglycosilated by the appropriate bacterial strains. Herbacetin diglucoside is part of the lignan macromolecule found in flaxseed (Struijs, Vincken, Verhoef, van Oostveen-van Casteren, Voragen, \& Gruppen, 2007). Accordingly, this compound was found in the flaxseed and soy mixture. Several $B$. breve and $B$. pseudocatenulatum strains were able to produce herbacetin as a result of the deglycosidation of herbacetin diglucoside, in a range of concentrations between 21 and $33 \mathrm{mg}$ of herbacetin per gram of flaxseed extract. Moreover, the flavonol aglycones quercetin and quercetagetin were produce mainly by B. breve and B. pseudocatenulatum strains. A possible explanation could be a common enzyme responsible for the deglycosilation of the glycosides of the three flavonols. This is, to our knowledge, the first time that the bacterial production of herbacetin and quercetagetin is described.

The production of quercetin by bacteria commonly found in food, as lactic acid bacteria and bifidobacteria, has been described in two Enterococcus strains (Schneider, Schwiertz, Collins, \& Blaut, 1999; Shin, Moon, Shin, Li, Lee, Kim, et al., 2016). In our work, just two B. breve and two B. pseudocatenulatum were able to produce quercetin, although a decrease on the glycoside isoquercetin was observed also for other Bifidobacterium strains. Interestingly, a collection of Bifidobacterium strains tested for rutin deglycosilation showed that any of the strains was able to produce quercetin, not even the $B$. pseudocatenulatum strains that were able to deglycosilate hesperidin into hesperetin (Amaretti, Raimondi, Leonardi, Quartieri, \& Rossi, 2015).

The simultaneous production of quercetin and kaempferol was carried out by Lactobacillus plantarum SDL 1413 from flavonoid glycosides (Lee, Oh, \& Jeong, 
327 2015). Moreover, Bifidobacterium strains have been described as capable of 328 transforming kaempferol glycoside into its aglycone form (Marotti, Bonetti, Biavati, 329 Catizone, \& Dinelli, 2007). Accordingly, we found increases on kaempferol 330 concentration in around half of the broths enriched with flaxseed and soy extracts and 331 fermented by Bifidobacterium strains. Again, the highest concentrations were achieved 332 by $B$. breve and $B$. pseudocatenulatum strains, together with $B$. dentium INIA P886.

333 Naringenin was produce by the majority of the Bifidobacterium strains, with higher 334 figures in $B$. breve, B. pseudocatenulatum, B. catenulatum and B. dentium strains. 335 Regarding the bacterial formation of this aglycone, it has been described in soybean 336 flour fermented with Lb. plantarum (Landete, et al., 2015).

337 Not all the aglycones studied were detected in the fermented broths. The lack of 338 detection of hesperetin, catechin, epicatechin, mirecitin and taxifolin may be due to the 339 absence of glycosidated precursors susceptible of being transformed by the glycosidases 340 of the Bifidobacterium strains tested.

341 In spite of the difference in the profile of aglycones produced, the differences in antioxidant activity and phenolic compounds were not as high as it could have been expected. Nevertheless, the higher antioxidant activity was measured in some of the bifidobacterial strains showing the higher production of flavonoid aglycones, i.e. $B$. breve INIA P367, B. breve INIA P776 and B. dentium INIA P883, although the high aglycone content of other bifidobacteria-fermented samples did not have a reflection in the antioxidant figures. Something similar happened with the determination of phenolic compounds, which did not correlate with the quantified aglycones, although higher 349 levels were achieved again by $B$. breve INIA P367, as well as by $B$. catenulatum INIA 350 P825. The lack of correlation between this two measurements and the profile of 351 compounds detected by HPLC-ESI/MS could be due the inability to identify and 
352 quantify all the phenolic compounds present in the samples. The composition in 353 glycosides of flaxseed and soy is complex, with presence of many different O- and C354 glycosides. Glycosylation attenuated the antioxidant activity and therefore the 355 deglycosylation reactions produce an increase in this activity (Cavia-Saiz, Busto, Pilar356 Izquierdo, Ortega, Pérez-Mateos, \& Muniz, 2010; Lee, et al., 2015). Nevertheless, it has 357 been described that the antioxidant activity of C-glucosides must be taken into account, 358 as it could be higher than that of the O-glycosides (Xiao, 2017). Those C-glycosides are 359 also less easily transformed into aglycones by the action of bacteria (Gaya, Peirotén, \& 360 Landete, 2017).

361 The previously described ability of Bifidobacterium strains to deglycosilate lignans and 362 isoflavones from flaxseed and soy extracts has been confirmed in this work in a mixture 363 of both extracts. Moreover, the production of other flavonoid aglycones has been 364 observed, with an important role of B. breve and B. pseudocatenulatum strains. One of 365 them, B. pseudocatenulatum INIA P815, which showed good levels of almost of the 366 aglycones studied, had shown previously the ability to produce urolithins A and B that 367 are phytoestrogens of interest in human health (Gaya, Peirotén, Medina, Álvarez, \& 368 Landete, 2018).

\section{Conclusion}

Besides isoflavones, lignans, flavonoids such as herbacetin, naringenin and kaempferol are also present in flaxseed and soy. The lignan and flavonoid aglycones have a high nutritional and health interest due to their increased bioactivity compared with the glycosides. Bifidobacterium is a genus with high glycosidase activity, in this work we 
376 show how that activity extents to several flavonoid compounds producing a wide

377 variety of flavonoid aglycones, some of them never described before as bacterial 378 products (herbacetin and quercetagetin). The B. breve and B. pseudocatenulatum strains 379 tested in this study stood out, showing high levels of SDG and isoflavones 380 deglycosilation alongside with a wide range of other flavonoids deglycosilated. Thus, 381 the use of these bacteria as probiotics, or in the fermentation of foods rich in flavonoids 382 and lignans, has a nutritional and health interest. 


\section{Compliance with Ethical Standards}

386

387 Funding: This study was funded by RTA2017-00002-00-00 from the Spanish Ministry 388 of Science, Innovation and Universities. We are grateful to the ICTAN (Institute of 389 Food Science, Technology and Nutrition, Madrid, Spain) Analysis Services Unit for 390 providing chromatography and mass spectrometry facilities. Authors want to thank the 391 authors to Ana del Olmo for her support in the analysis of antioxidant activity.

393 Compliance with ethical standards. This article does not contain any studies with 394 human participants or animals performed by any of the authors.

396 Conflicts of Interest: Authors declare no conflict of interest. 
398

399

400

401

402

403

404

405

406

407

408

409

410

411

412

413

414

415

416

417

418

419

420

421

422

423

\section{References}

Airoldi, C., La Ferla, B., Orazio, G., Ciaramelli, C. \& Palmioli, A. (2018). Flavonoids in the treatment of Alzheimer's and other neurodegenerative diseases. Current Medicinal Chemistry, 25, 3228-3246.

Amaretti, A., Raimondi, S., Leonardi, A., Quartieri, A. \& Rossi, M. (2015). Hydrolysis of the rutinose-conjugates flavonoids rutin and hesperidin by the gut microbiota and bifidobacteria. Nutrients, 7, 2788-2800.

Cavia-Saiz, M., Busto, M. D., Pilar-Izquierdo, M. C., Ortega, N., Pérez-Mateos, M. \& Muniz, P. (2010). Antioxidant properties, radical scavenging activity and biomolecule protection capacity of flavonoid naringenin and its glycoside naringin: a comparative study. Journal of the Science of Food and Agriculture, 90, 1238-1244.

Correa, C. R., Li, L., Aldini, G., Carini, M., Oliver Chen, C. Y., Chun, H.-K., Cho, S.-M., Park, K.-M., Russell, R. M., Blumberg, J. B. \& Yeum, K.-J. (2010). Composition and stability of phytochemicals in five varieties of black soybeans (Glycine max). Food Chemistry, 123, 1176-1184.

del Olmo, A., Picón, A. \& Núñez, M. (2018). Cheese supplementation with five species of edible seaweeds: Effect on microbiota, antioxidant activity, colour, texture and sensory characteristics. International Dairy Journal, 84, 36-45.

Devi, K. P., Malar, D. S., Nabavi, S. F., Sureda, A., Xiao, J., Nabavi, S. M. \& Daglia, M. (2015). Kaempferol and inflammation: From chemistry to medicine. Pharmacological Research, 99, 1-10.

Egert, S., Bosy-Westphal, A., Seiberl, J., Kürbitz, C., Settler, U., Plachta-Danielzik, S., Wagner, A. E., Frank, J., Schrezenmeir, J., Rimbach, G., Wolffram, S. \& Müller, M. J. (2009). Quercetin reduces systolic blood pressure and plasma oxidised low-density lipoprotein concentrations in overweight subjects with a high-cardiovascular disease risk phenotype: a 
double-blinded, placebo-controlled cross-over study. British Journal of Nutrition, 102, 10651074.

Ferraces-Casais, P., Lage-Yusty, M. A., Rodríguez-Bernaldo de Quirós, A. \& LópezHernández, J. (2012). Evaluation of bioactive compounds in fresh edible seaweeds. Food Analytical Methods, 5, 828-834.

Gaya, P., Arqués, J. L., Medina, M., Álvarez, I. \& Landete, J. M. (2016a). A new HPLCPAD/HPLC-ESI-MS method for the analysis of phytoestrogens produced by bacterial metabolism. Food Analytical Methods, 9, 537-547.

Gaya, P., Peirotén, Á., Álvarez, I., Medina, M. \& Landete, J. M. (2018). Production of the bioactive isoflavone $O$-desmethylangolensin by Enterococcus faecium INIA P553 with high efficiency. Journal of Functional Foods, 40, 180-186.

Gaya, P., Peirotén, Á. \& Landete, J. M. (2017). Transformation of plant isoflavones into bioactive isoflavones by lactic acid bacteria and bifidobacteria. Journal of Functional Foods, 39, 198-205.

Gaya, P., Peirotén, Á., Medina, M., Álvarez, I. \& Landete, J. M. (2018). Bifidobacterium pseudocatenulatum INIA P815: The first bacterium able to produce urolithins A and B from ellagic acid. Journal of Functional Foods, 45, 95-99.

Gaya, P., Peirotén, Á., Medina, M. \& Landete, J. M. (2016b). Isoflavone metabolism by a collection of lactic acid bacteria and bifidobacteria with biotechnological interest. International Journal of Food Sciences and Nutrition, 67, 117-124.

Goyal, A., Sharma, V., Upadhyay, N., Gill, S. \& Sihag, M. (2014). Flax and flaxseed oil: an ancient medicine \& modern functional food. Journal of Food Science and Technology, 51, $1633-1653$.

Guadamuro, L., Flórez, A. B., Alegría, Á., Vázquez, L. \& Mayo, B. (2017). Characterization of four $\beta$-glucosidases acting on isoflavone-glycosides from Bifidobacterium pseudocatenulatum IPLA 36007. Food Research International, 100, 522-528.

Herchi, W., Arraez-Roman, D., Trabelsi, H., Bouali, I., Boukhchina, S., Kallel, H., SeguraCarretero, A. \& Fernández-Gutiérrez, A. (2014). Phenolic compounds in flaxseed: a review 

of their properties and analytical methods. An overview of the last decade. Journal of Oleo Science, 63, 7-14.

Landete, J. M., Arques, J., Medina, M., Gaya, P., de Las Rivas, B. \& Muñoz, R. (2016). Bioactivation of phytoestrogens: Intestinal bacteria and health. Critical Reviews in Food Science and Nutrition, 56, 1826-1843.

Landete, J. M., Hernández, T., Robredo, S., Dueñas, M., de las Rivas, B., Estrella, I. \& Muñoz, R. (2015). Effect of soaking and fermentation on content of phenolic compounds of soybean (Glycine max cv. Merit) and mung beans (Vigna radiata L Wilczek). International Journal of Food Sciences and Nutrition, 66, 203-209.

Lee, Y., Oh, J. \& Jeong, Y.-S. (2015). Lactobacillus plantarum-mediated conversion of flavonoid glycosides into flavonols, quercetin, and kaempferol in Cudrania tricuspidata leaves. Food Science and Biotechnology, 24, 1817-1821.

Lou, H., Jing, X., Ren, D., Wei, X. \& Zhang, X. (2012). Eriodictyol protects against H2O2induced neuron-like PC12 cell death through activation of Nrf2/ARE signaling pathway. Neurochemistry International, 61, 251-257.

Marotti, I., Bonetti, A., Biavati, B., Catizone, P. \& Dinelli, G. (2007). Biotransformation of common bean (Phaseolus vulgaris L.) flavonoid glycosides by Bifidobacterium species from human intestinal origin. Journal of Agricultural and Food Chemistry, 55(10), 3913-3919.

McClure, T., Cocuron, J.-C., Osmark, V., McHale, L. K., \& Alonso, A. P. (2017). Impact of environment on the biomass composition of soybean (Glycine max) seeds. Journal of Agricultural and Food Chemistry, 65, 6753-6761.

Narváez-Mastache, J. M., Novillo, F. \& Delgado, G. (2008). Antioxidant aryl-prenylcoumarin, flavan-3-ols and flavonoids from Eysenhardtia subcoriacea. Phytochemistry, 69, 451-456.

Oomah, B. D. (2001). Flaxseed as a functional food source. Journal of the Science of Food and Agriculture, 81, 889-894.

Peirotén, A., Arqués, J. L., Medina, M. \& Rodríguez-Mínguez, E. (2018). Bifidobacterial strains shared by mother and child as source of probiotics. Beneficial Microbes, 9, 231-238. 
479 Peirotén, Á., Bravo, D. \& Landete, J. M. (2019a). Bacterial metabolism as responsible of 480 beneficial effects of phytoestrogens on human health. Critical Reviews in Food Science and $481 \quad$ Nutrition, 1-16. doi: 10.1080/10408398.2019.1622505.

482 Peirotén, Á., Gaya, P., Álvarez, I., Bravo, D. \& Landete, J. M. (2019b). Influence of different 483 lignan compounds on enterolignan production by Bifidobacterium and Lactobacillus strains. $484 \quad$ International Journal of Food Microbiology, 289, 17-23.

485 Rietjens, I. M. C. M., Louisse, J. \& Beekmann, K. (2017). The potential health effects of dietary 486 phytoestrogens. British Journal of Pharmacology, 174, 1263-1280.

487 Rodríguez, E., Arqués, J. L., Rodríguez, R., Peirotén, A., Landete, J. M. \& Medina, M. (2012). 488 Antimicrobial properties of probiotic strains isolated from breast-fed infants. Journal of $489 \quad$ Functional Foods, 4, 542-551.

490 Schneider, H., Schwiertz, A., Collins, M. D. \& Blaut, M. (1999). Anaerobic transformation of 491 quercetin-3-glucoside by bacteria from the human intestinal tract. Archives of Microbiology, 17, 81-91.

Shin, N. R., Moon, J. S., Shin, S. Y., Li, L., Lee, Y. B., Kim, T. J. \& Han, N. S. (2016). 494 Isolation and characterization of human intestinal Enterococcus avium EFEL009 converting rutin to quercetin. Letters in Applied Microbiology, 62, 68-74.

Sirotkin, A. V., \& Harrath, A. H. (2014). Phytoestrogens and their effects. European Journal of Pharmacology, 741, 230-236.

Struijs, K., Vincken, J. P., Verhoef, R., van Oostveen-van Casteren, W. H., Voragen, A. G. \& Gruppen, H. (2007). The flavonoid herbacetin diglucoside as a constituent of the lignan macromolecule from flaxseed hulls. Phytochemistry, 68, 1227-1235.

501 Toure, A. \& Xu, X. (2010). Flaxseed lignans: Source, biosynthesis, metabolism, antioxidant 502 activity, bio-active components, and health benefits. Comprehensive Reviews in Food $503 \quad$ Science and Food Safety, 9, 261-269.

504 Vaya, J. \& Tamir, S. (2004). The relation between the chemical structure of flavonoids and their 505 estrogen-like activities. Current Medicinal Chemistry, 11, 1333-1343. 
506 Veeramani, C., Alsaif, M. A., \& Al-Numair, K. S. (2018). Herbacetin, a flaxseed flavonoid, 507 ameliorates high percent dietary fat induced insulin resistance and lipid accumulation 508 through the regulation of hepatic lipid metabolizing and lipid-regulating enzymes. Chemico509 Biological Interactions, 288, 49-56.

510 Venkateswara, R. P., Kiran, S., Rohini, P. \& Bhagyasree, P. (2017). Flavonoid: A review on 511 naringenin. Journal of Pharmacognosy and Phytochemistry, 6, 2778-2783.

512 Wang, W., Xu, H., Chen, H., Tai, K., Liu, F. \& Gao, Y. (2016). In vitro antioxidant, anti513 diabetic and antilipemic potentials of quercetagetin extracted from marigold (Tagetes erecta 514 L.) inflorescence residues. Journal of Food Science and Technology, 53, 2614-2624.

515 Xiao, J. (2017). Dietary flavonoid aglycones and their glycosides: Which show better biological $516 \quad$ significance? Critical Reviews in Food Science and Nutrition, 57, 1874-1905.

517 Zamora-Ros, R., Touillaud, M., Rothwell, J. A., Romieu, I. \& Scalbert, A. (2014). Measuring 518 exposure to the polyphenol metabolome in observational epidemiologic studies: current tools 519 and applications and their limits. American Journal of Clinical Nutrition, 100, 11-26.

520

521 
522 Table 1. Bifidobacterium strains tested in this work.

\begin{tabular}{|c|c|c|}
\hline Strain & Origin & Reference \\
\hline B. adolescentis INIA P549 & breast-fed infant faeces & Rodríguez et al. 2012 \\
\hline B. adolescentis INIA P659 & breast-fed infant faeces & Rodríguez et al. 2012 \\
\hline B. animalis INIA P490 & breast-fed infant faeces & Rodríguez et al. 2012 \\
\hline B. animalis INIA P913 & adult faeces & INIA culture collection \\
\hline B. bifidum INIA P466 & breast-fed infant faeces & Rodríguez et al. 2012 \\
\hline B. bifidum INIA P475 & breast-fed infant faeces & Rodríguez et al. 2012 \\
\hline B. breve INIA 367 & breast-fed infant faeces & Rodríguez et al. 2012 \\
\hline B. breve INIA P12 & breast-fed infant faeces & Rodríguez et al. 2012 \\
\hline B. breve INIA P776 & child faeces & Peirotén et al. 2018 \\
\hline B. catenulatum INIA P732 & breast-fed infant faeces & Peirotén et al. 2018 \\
\hline B. catenulatum INIA P765 & adult faeces & Peirotén et al. 2018 \\
\hline B. catenulatum INIA P825 & child faeces & Peirotén et al. 2018 \\
\hline B. dentium INIA P883 & adult faeces & INIA culture collection \\
\hline B. dentium INIA P886 & adult faeces & INIA culture collection \\
\hline B. infantis INIA P593 & breast-fed infant faeces & Rodríguez et al. 2012 \\
\hline B. infantis INIA P722 & breast-fed infant faeces & Peirotén et al. 2018 \\
\hline B. infantis INIA P728 & breast-fed infant faeces & Peirotén et al. 2018 \\
\hline B. longum INIA P750 & child faeces & Peirotén et al. 2018 \\
\hline B. longum INIA P9 & breast-fed infant faeces & Rodríguez et al. 2012 \\
\hline
\end{tabular}




\begin{tabular}{lll} 
B. pseudocatenulatum INIA P815 & adult faeces & INIA culture collection \\
B. pseudocatenulatum INIA P846 & child faeces & Peirotén et al. 2018 \\
$\begin{array}{l}\text { B. pseudocatenulatum INIA P946 } \\
\text { B. pseudolongum INIA P2 }\end{array}$ & breast-fed infant faeces & INIA culture collection \\
B. pseudolongum INIA P120 & breast-fed infant faeces & Rodríguez et al. 2012 \\
B. pseudolongum INIA P977 & breast-fed infant faeces & Rodríguez et al. 2012 \\
\hline
\end{tabular}


525 Table 2. Secoisolariciresinol (SECO) and isoflavone aglycones (mg per liter of fermentation media) produced from flaxseed and soy extracts 526 after incubation with a collection of Bifidobacterium strains.

\begin{tabular}{lccc}
\hline & Lignans & \multicolumn{2}{c}{ Isoflavones } \\
& SECO & Daidzein & Genistein \\
\hline Control & $3.9 \pm 0.4$ & $27.9 \pm 2.3$ & $38.6 \pm 12.6$ \\
B. adolescentis INIA P549 & $3.8 \pm 0.5$ & $58.2 \pm 7.7$ & $62.5 \pm 21.3$ \\
B. adolescentis INIA P659 & $5.8 \pm 2.1$ & $28.9 \pm 5.6$ & $57.2 \pm 12.2$ \\
B. animalis INIA P490 & $74.6 \pm 13.3$ & $82.3 \pm 9.7$ & $101.2 \pm 7.8$ \\
B. animalis INIA P913 & $33.5 \pm 17.6$ & $99.2 \pm 24.5$ & $156.2 \pm 15.8$ \\
B. bifidum INIA P466 & $34.2 \pm 1.3$ & $131.6 \pm 3.3$ & $166.5 \pm 21.3$ \\
B. bifidum INIA P475 & $9.4 \pm 2.3$ & $49.2 \pm 14.2$ & $72.4 \pm 8.7$ \\
B. breve INIA 367 & $116.4 \pm 13.7$ & $187.3 \pm 10.7$ & $221.8 \pm 9.7$ \\
B. breve INIA P12 & $81.3 \pm 4.5$ & $172.3 \pm 31.2$ & $210.2 \pm 23.1$ \\
B. breve INIA P776 & $148.9 \pm 16.8$ & $181.8 \pm 17.1$ & $205.6 \pm 21.2$ \\
B. catenulatum INIA P732 & $41.3 \pm 12.6$ & $147.3 \pm 27.2$ & $103.4 \pm 33.5$ \\
B. catenulatum INIA P765 & $2.9 \pm 2.1$ & $34.7 \pm 7.6$ & $61.2 \pm 8.1$ \\
B. catenulatum INIA P825 & $42.3 \pm 3.6$ & $67.2 \pm 21.8$ & $206.5 \pm 8.9$ \\
B. dentium INIA P883 & $54.6 \pm 6.5$ & $141.2 \pm 5.3$ & $166.4 \pm 22.4$ \\
B. dentium INIA P886 & $18.9 \pm 8.5$ & $107.6 \pm 20.0$ & $142.3 \pm 9.3$ \\
B. infantis INIA P593 & $3.3 \pm 0.8$ & $51.6 \pm 12.5$ & $59.1 \pm 9.1$ \\
\hline
\end{tabular}




\begin{tabular}{lccc} 
B. infantis INIA P722 & $4.3 \pm 1.1$ & $23.3 \pm 1.9$ & $43.3 \pm 2.9$ \\
B. infantis INIA P728 & $7.7 \pm 1.5$ & $57.3 \pm 13.2$ & $112.4 \pm 4.6$ \\
B. longum INIA P750 & $12.0 \pm 0.9$ & $47.8 \pm 14.5$ & $98.7 \pm 18.5$ \\
B. longum INIA P9 & $5.8 \pm 0.7$ & $40.3 \pm 6.7$ & $91.2 \pm 8.2$ \\
B. pseudocatenulatum INIA P815 & $102.6 \pm 57.6$ & $139.4 \pm 32.4$ & $141.5 \pm 21.7$ \\
B. pseudocatenulatum INIA P846 & $152.8 \pm 22.1$ & $158.4 \pm 12.0$ & $168.2 \pm 18.8$ \\
B. pseudocatenulatum INIA P946 & $204.2 \pm 35.3$ & $154.8 \pm 6.4$ & $195.7 \pm 11.1$ \\
B. pseudolongum INIA P2 & $13.6 \pm 4.4$ & $65.4 \pm 2.3$ & $73.2 \pm 15.2$ \\
B. pseudolongum INIA P120 & $16.8 \pm 7.5$ & $46.7 \pm 2.6$ & $69.5 \pm 11.7$ \\
B. pseudolongum INIA P977 & $15.8 \pm 4.4$ & $62.1 \pm 16.9$ & $101.6 \pm 8.0$ \\
\hline
\end{tabular}

527 
529 Table 3. Flavanones, flavones and flavonols ( $\mathrm{mg}$ per liter of fermentation media) produced from flaxseed and soy extracts after incubation with a 530 collection of Bifidobacterium strains.

\begin{tabular}{|c|c|c|c|c|c|c|c|c|}
\hline & \multicolumn{2}{|c|}{ Flavanones } & \multicolumn{2}{|c|}{ Flavones } & \multicolumn{4}{|c|}{ Flavonols } \\
\hline & Naringenin & Eriodyctiol & Luteolin & Apigenin & Herbacetin & Kaempferol & Quercetin & Quercetagetin \\
\hline Control & $0.87 \pm 0.06$ & $0.29 \pm 0.03$ & $0.76 \pm 0.06$ & n.d. & n.d. & $0.19 \pm 0.04$ & n.d. & n.d. \\
\hline B. adolescentis INIA P549 & $0.31 \pm 0.11$ & $0.09 \pm 0.04$ & $0.81 \pm 0.21$ & n.d. & n.d. & $0.08 \pm 0.04$ & n.d. & n.d. \\
\hline B. adolescentis INIA P659 & $2.62 \pm 0.17$ & $0.39 \pm 0.05$ & $0.79 \pm 0.41$ & $0.22 \pm 0.16$ & $0.04 \pm 0.02$ & $0.19 \pm 0.11$ & n.d. & n.d. \\
\hline B. animalis INIA P490 & $2.70 \pm 0.71$ & $0.41 \pm 0.03$ & $1.16 \pm 0.32$ & $0.33 \pm 0.09$ & $0.57 \pm 0.07$ & $0.71 \pm 0.09$ & n.d. & n.d. \\
\hline B. animalis INIA P913 & $4.09 \pm 0.71$ & $0.45 \pm 0.21$ & $0.89 \pm 0.13$ & $0.40 \pm 0.06$ & $1.87 \pm 0.43$ & $0.87 \pm 0.32$ & n.d. & n.d. \\
\hline B. bifidum INIA P466 & $1.90 \pm 0.08$ & $0.31 \pm 0.06$ & $1.04 \pm 0.34$ & $0.19 \pm 0.01$ & n.d. & $0.28 \pm 0.03$ & n.d. & n.d. \\
\hline B. bifidum INIA P475 & $0.78 \pm 0.23$ & $0.34 \pm 0.11$ & $0.90 \pm 0.06$ & $0.07 \pm 0.02$ & n.d. & $0.12 \pm 0.01$ & n.d. & n.d. \\
\hline B. breve INIA 367 & $4.13 \pm 0.56$ & $0.52 \pm 0.02$ & $0.98 \pm 0.18$ & $0.27 \pm 0.03$ & $57.37 \pm 6.73$ & $2.00 \pm 0.56$ & $0.90 \pm 0.08$ & $0.93 \pm 0.08$ \\
\hline B. breve INIA P12 & $4.33 \pm 1.01$ & $0.50 \pm 0.13$ & $1.15 \pm 0.07$ & $0.55 \pm 0.21$ & $42.67 \pm 8.09$ & $2.29 \pm 0.34$ & n.d. & $0.63 \pm 0.17$ \\
\hline B. breve INIA P776 & $4.11 \pm 0.38$ & $0.56 \pm 0.13$ & $0.96 \pm 0.10$ & $0.46 \pm 0.02$ & $54.30 \pm 13.81$ & $2.30 \pm 0.08$ & $0.95 \pm 0.19$ & $0.53 \pm 0.05$ \\
\hline B. catenulatum INIA P732 & $3.97 \pm 0.70$ & $0.50 \pm 0.11$ & $1.06 \pm 0.11$ & $0.30 \pm 0.03$ & $0.02 \pm 0.01$ & $0.47 \pm 0.08$ & n.d. & n.d. \\
\hline B. catenulatum INIA P765 & $4.41 \pm 0.30$ & $0.58 \pm 0.02$ & $0.93 \pm 0.36$ & $0.36 \pm 0.12$ & n.d. & $0.41 \pm 0.11$ & n.d. & n.d. \\
\hline B. catenulatum INIA P825 & $4.35 \pm 0.22$ & $0.61 \pm 0.15$ & $0.77 \pm 0.02$ & $0.29 \pm 0.15$ & $0.04 \pm 0.03$ & $0.45 \pm 0.01$ & n.d. & n.d. \\
\hline B. dentium INIA P883 & $3.56 \pm 0.15$ & $0.46 \pm 0.08$ & $0.72 \pm 0.32$ & n.d. & n.d. & $0.28 \pm 0.05$ & n.d. & n.d. \\
\hline B. infantis INIA P593 & $2.57 \pm 0.33$ & $0.38 \pm 0.07$ & $0.72 \pm 0.17$ & $0.18 \pm 0.011$ & $0.03 \pm 0.01$ & $0.21 \pm 0.05$ & n.d. & n.d. \\
\hline B. infantis INIA P722 & $3.02 \pm 0.09$ & $0.46 \pm 0.13$ & $0.74 \pm 0.05$ & $0.17 \pm 0.03$ & n.d. & $0.19 \pm 0.15$ & n.d. & n.d. \\
\hline B. infantis INIA P728 & $3.24 \pm 1.41$ & $0.50 \pm 0.21$ & $0.14 \pm 0.02$ & $0.13 \pm 0.02$ & n.d. & $0.13 \pm 0.10$ & n.d. & n.d. \\
\hline B. longum INIA P750 & $2.86 \pm 0.43$ & $0.45 \pm 0.08$ & $0.94 \pm 0.08$ & $0.49 \pm 0.16$ & $0.02 \pm 0.01$ & $0.21 \pm 0.02$ & n.d. & n.d. \\
\hline B. longum INIA P9 & $0.88 \pm 0.09$ & $0.35 \pm 0.08$ & $0.95 \pm 0.11$ & n.d. & n.d. & $0.08 \pm 0.03$ & n.d. & n.d. \\
\hline B. pseudocatenulatum INIA P815 & $4.21 \pm 0.08$ & $0.62 \pm 0.05$ & $1.15 \pm 0.07$ & $0.33 \pm 0.09$ & $64.74 \pm 5.62$ & $2.36 \pm 0.41$ & $0.98 \pm 0.07$ & $1.12 \pm 0.32$ \\
\hline B. pseudocatenulatum INIA P846 & $4.41 \pm 0.65$ & $0.60 \pm 0.01$ & $0.97 \pm 0.19$ & $0.50 \pm 0.04$ & $0.78 \pm 0.19$ & $0.88 \pm 0.18$ & n.d. & n.d. \\
\hline B. pseudocatenulatum INIA P946 & $4.38 \pm 0.21$ & $0.70 \pm 0.43$ & $0.86 \pm 0.07$ & $0.48 \pm 0.010$ & $56.90 \pm 7.81$ & $1.77 \pm 0.05$ & $0.97 \pm 0.31$ & $1.44 \pm 0.16$ \\
\hline
\end{tabular}


Page 29 of 34

1
2
3
4
5
6
7
8
9
10
11
12
13
14
15
16
17
18
19
20
21
22
23
24
25
26
27
28
29
30
31
32
33
34
35
36
37
38
39
40
41
42
43
44
45
46

532

533

534
International Journal of Food Science \& Technology

$\begin{array}{llllrrrrr}\text { B. pseudolongum INIA P120 } & 3.01 \pm 0.17 & 0.49 \pm 0.06 & 0.90 \pm 0.21 & 0.40 \pm 0.08 & \text { n.d. } & 0.26 \pm 0.21 & \text { n.d. } & \text { n.d. } \\ \text { B. pseudolongum INIA P2 } & 2.09 \pm 0.21 & 0.41 \pm 0.11 & 0.84 \pm 0.16 & \text { n.d. } & 0.03 \pm 0.01 & 0.17 \pm 0.05 & \text { n.d. } & \text { n.d. } \\ \text { B. pseudolongum INIA P977 } & 0.94 \pm 0.16 & 0.48 \pm 0.17 & 0.80 \pm 0.05 & \text { n.d. } & \text { n.d. } & 0.14 \pm 0.08 & \text { n.d. } & \text { n.d. }\end{array}$


537 Figure 1. HPLC-ESI (A) and HPLC-ESI-MS/MS (B, C) analyses to identify herbacetin and quercetin produced by Bifidobacterium. 538

539 Figure 2. HPLC-ESI (A) and HPLC-ESI-MS/MS (B) analyses to identify quercetagetin produced by Bifidobacterium. 
$542 \quad$ Figure 1

543

x10 2 -ESI EIC(301.0354) Scan Frag=120.0V Muestra G14 25ul metodo ICTAN.d

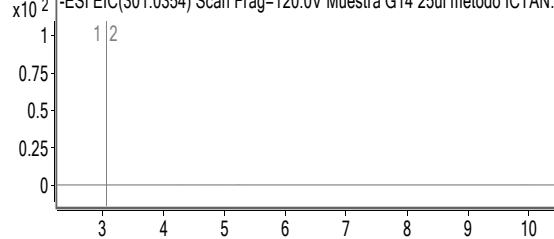

Herbacetin

ESI EIC(301.0354)

19,0
362594187.12

Quercetin

$\operatorname{Tr} 19.0$

ESI EIC(301.0354) 50819450.00 Tr 21.0

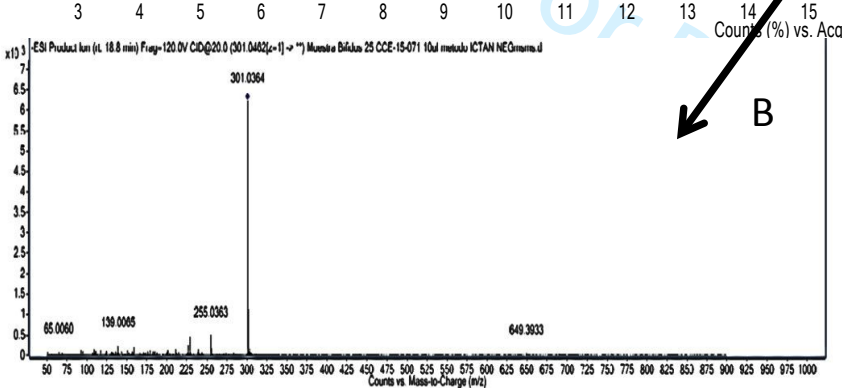

A

A
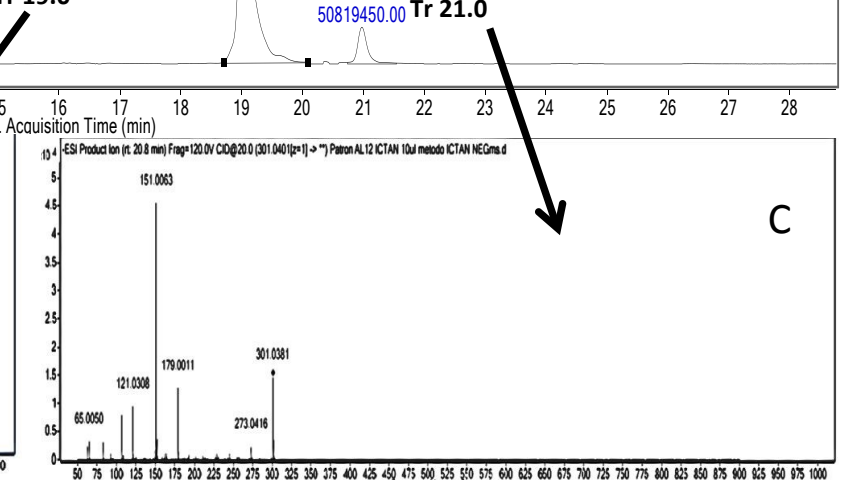

Institute of Food Science and Technology 


\section{Figure 2}
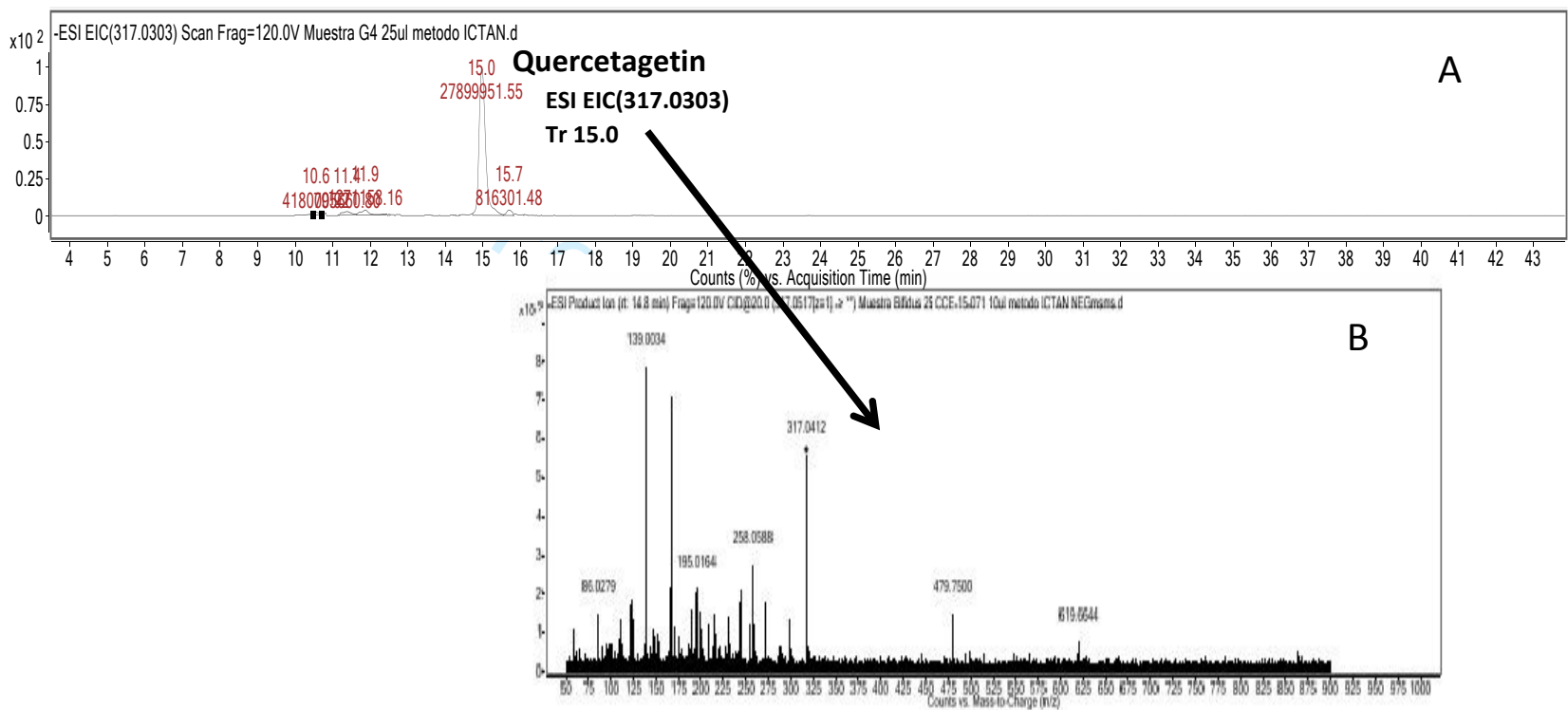

546 


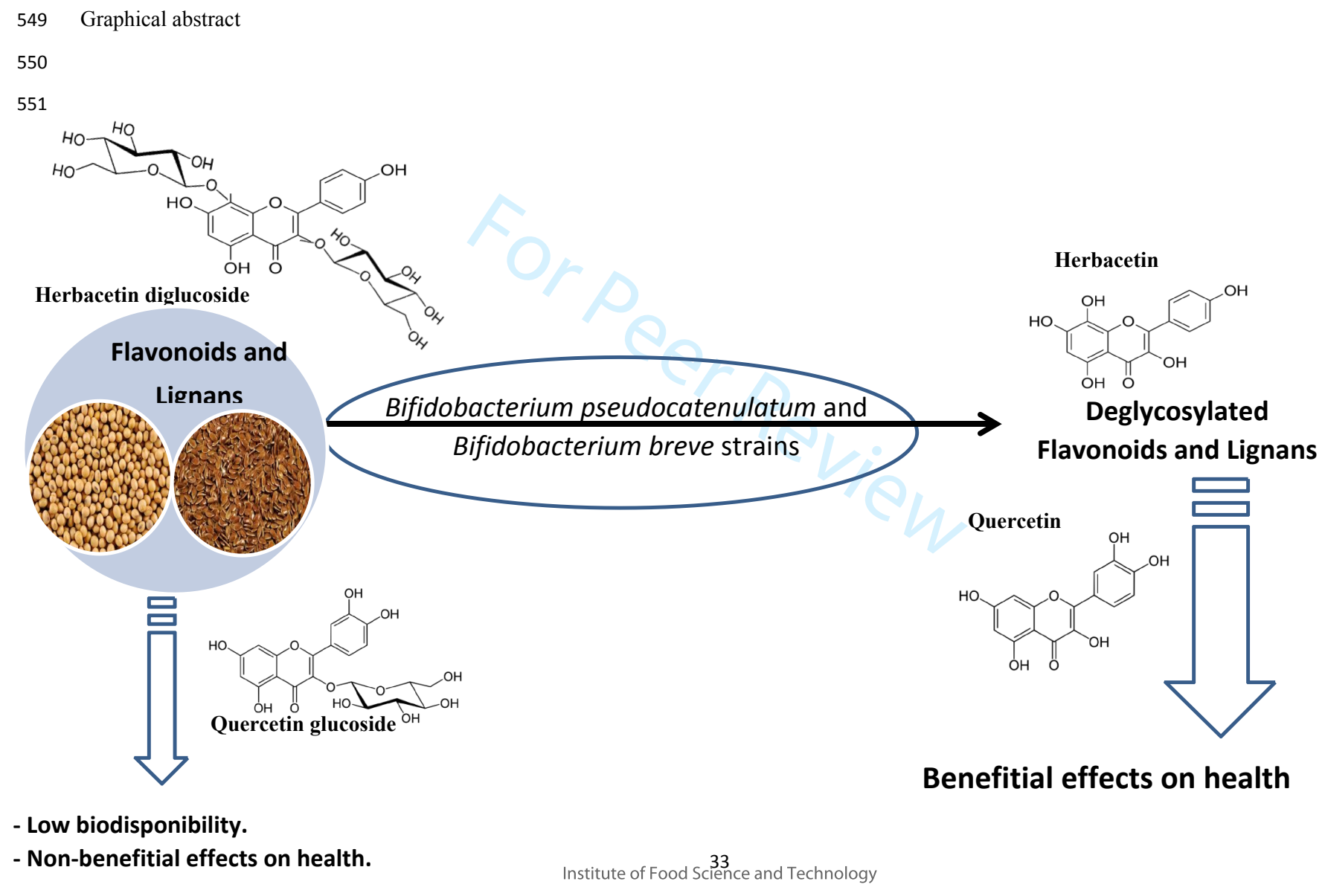


Figure 1. HPLC-ESI chromatograms of flavonoid aglycones produced by Bifidobacterium strains.
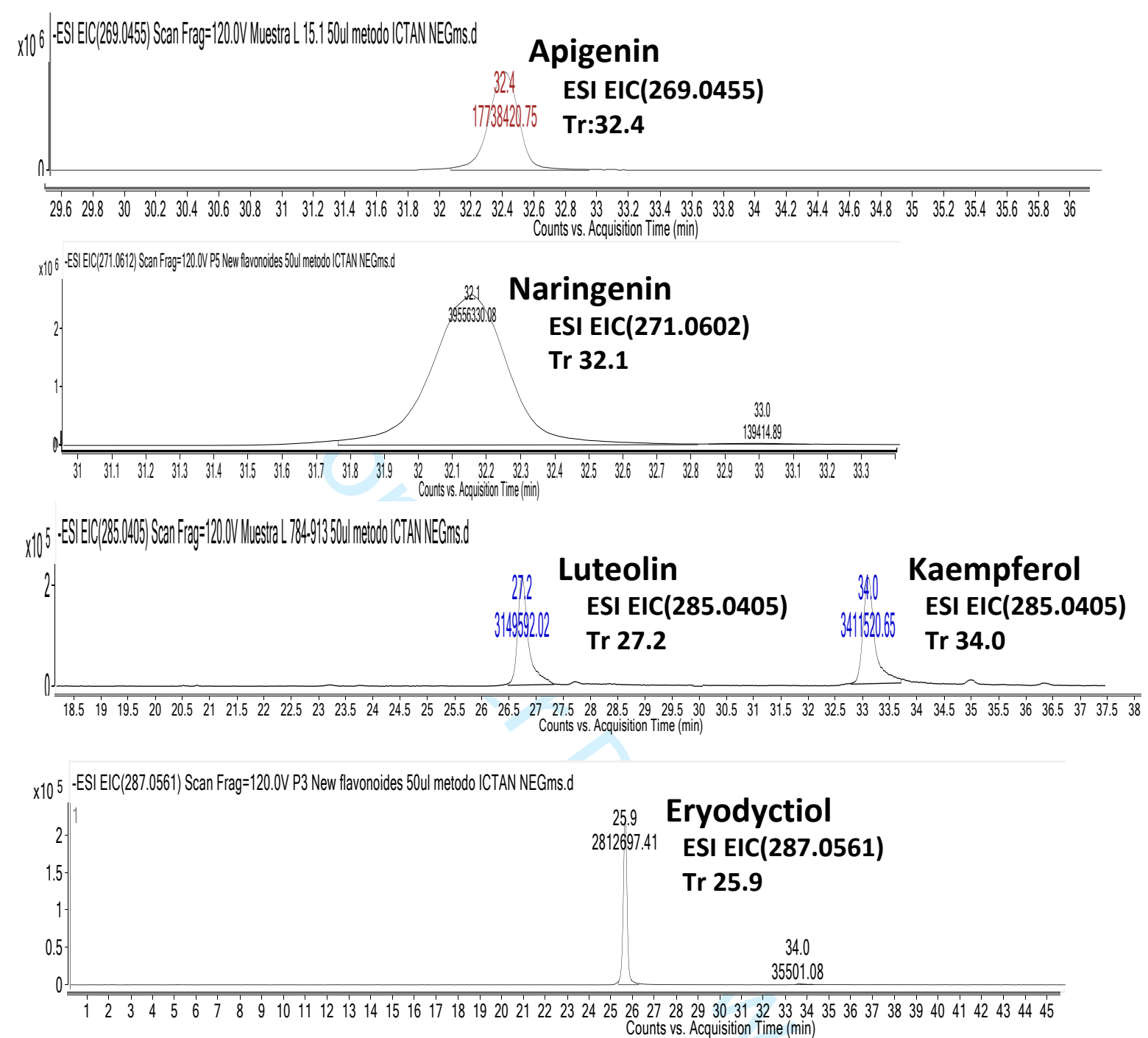